\title{
Development and Deployment of the Windbridge Psi and Related Phenomena Awareness Questionnaire (WPRPAQ)
}

\author{
Julie Beischel \\ Windbridge Research Center, Tucson, AZ, www.windbridge.org
}

MARK BoccuzzI

Windbridge Institute, Tucson, AZ, www.windbridgeinstitute.com

Submitted July 16, 2019; Accepted December 16, 2019; Published March 30, 2020

https://doi.org/10.31275/2020/1623

Creative Commons License CC-BY-NC

Abstract-Surveys regarding anomalous beliefs and exceptional experiences are an important methodology in sociology and psi-related research. Previously published questionnaires, however, contain various limitations in their philosophy, language, and usefulness. This study aimed to develop a psi survey and collect data from an experience-centered perspective. Established survey development and piloting methods were used to create the Windbridge Psi and Related Phenomena Awareness Questionnaire (WPRPAQ), a novel, 10-item, web-based instrument which phenomenologically describes experiential phenomena without using problematic terms and asks respondents to signify whether they are aware of the phenomena or not and, if so, what experience they have had with them. For analysis, WPRPAQ items were categorized as bidirectional which involve two or more people and can be given and/ or received (energy healing, mediumship, and telepathy) or unidirectional which generally involve only the experiencer/respondent (clairvoyance, micropsychokinesis [microPK] and macropsychokinesis [macroPK], out-of-body experiences, near-death experiences, children's memories of previous lives, and precognition). Online survey response data were collected from self-identified mediums $(n=316)$ and non-mediums $(n=$ 1,030) with no demographic statistical differences: $53.2 \pm 10.1$ and $53.9 \pm$ 11.9 years of age, respectively; $89.5 \%$ and $85.5 \%$ female, respectively; both roughly $95 \%$ white. More than $80 \%$ of each participant sample reported being aware of all 10 phenomena. The portion of mediums who reported 
awareness of microPK and macroPK was significantly larger than the portion of non-mediums for those phenomena (each $p<.00001)$. A significantly larger portion of mediums than non-mediums reported experiencing each of the 10 phenomena (all $p<.0001$ ). Ideally, the WPRPAQ can be used by other researchers to assess awareness of psi and related phenomena and the prevalence of those experiences in other populations.

Keywords: psi survey; microPK; macroPK; mediums; psi questionnaire; psi phenomena

Surveys regarding anomalous beliefs and experiences have regularly been an important methodology in sociology, parapsychology, and psi-related research, reviewed, for example, by Irwin (2009). These studies have included examinations of experiences in samples from the general population (e.g., Castro et al., 2014; Palmer, 1979) as well as samples from specific populations such as Association for Research and Enlightenment (ARE) members (Kohr, 1980) and Spiritualist mental mediums (Roxburgh \& Roe, 2011). Comparisons of both types of samples also have been done. Fach et al. (2013), for example, compared the experiences of a cross section of the general population to those of clients reporting exceptional experiences who actively sought advice from counselors at the Institut für Grenzgebiete der Psychologie und Psychohygiene (IGPP; Institute for Frontier Areas of Psychology and Mental Health). Often, the aim of these questionnaire studies is to examine relationships between experiences or beliefs and, for example, psychological factors (e.g., Rabeyron \& Watt, 2010), well-being (e.g., Kennedy et al., 1994), and trauma (e.g., Irwin, 1994).

Previously published questionnaires, however, contain various limitations; this extends beyond simple participant burden issues (i.e. large numbers of items). Goulding and Parker (2001) found that the use of psychometric instruments to measure psi-related abilities, beliefs, and/or experiences "is steered to a large extent by the underlying model or ideology concerning what psychic experiences are" (p. 73). They emphasized an "unabated" trend by researchers to focus on the dysfunctional aspects of psi-related experiences such as lack of critical thinking ability and proneness to psychosis in their instruments. In reality, these experiences are frequent within the general, non-clinical population and not usually associated with mental disorders (e.g., Moreira-Almeida \& Lotufo-Neto, 2017). 
Other more specific issues also exist. Several instruments use loaded/leading terminology (e.g., Otis \& Alcock, 1982). For example, the Paranormal Belief Scale (Tobacyk \& Milford, 1983) includes the item "Reincarnation does occur;" a term related to specific religious and spiritual worldviews. Similar issues arise with the widespread use of the terms soul, spirit, ghost, God, miracle, etc. The use of these types of terms is problematic as some may inhibit respondents from reporting the experience of interest (Moreira-Almeida \& Lotufo-Neto, 2017). This may be a general issue with instruments referring to relevant experiences as paranormal within the survey title (e.g., Randall, 1997, Tobacyk \& Milford, 1983), though modern research has shown that "the paranormal is (still) normal" (Castro et al., 2014, p. 1).

Some instruments use unclear language, making it potentially difficult to quantify one's agreement with a particular item. For example, the first item in the Paranormal Short Inventory (Randall, 1997) is: "It is probably true that certain people can predict the future quite accurately." The instrument asks respondents to strongly, somewhat, or slightly agree or disagree with the probable truth about the quite accurate abilities of others. Other items in this instrument ask respondents to quantify their agreement with items including "for the most part," "it is quite possible," and "as a general rule."

Terms with no associated definitions are regularly used in instruments (e.g., Van de Castle \& White, 1955; Randall, 1997). For example, the Anomalous Experiences Inventory (AEl; Gallagher et al., 1994) includes the items "I have had a psychic experience," "I believe in the unconscious," and "I believe that many paranormal occurrences are real." Similarly, the Australian Sheep-Goat Scale (ASGS; Thalbourne, 1995) uses the terms ESP, psychic, and telepathy without defining them (the term psychokinesis is defined within the ASGS).

Many instruments use what is now considered discriminatory or disparaging language (e.g., Nixon, 1925). For example, the Paranormal Belief Scale (Tobacyk \& Milford, 1983) includes items about belief in witches and voodoo, established international spiritual and religious practices (e.g., "Witches do exist") and combines these into a "Witchcraft" subscale. In the revised version of this instrument (Tobacyk, 2004), the items referencing voodoo have been changed to refer instead to formulas, incantations, and casting spells. 
Often surveys involve an interdependence of items (e.g., Gallagher et al., 1994). For example, the ASGS (Thalbourne, 1995) includes the items "I believe in life after death" and "I believe that some people can contact spirits of the dead" though the latter relies on the former being true.

Older instruments may contain items not widely relevant today. For example, Bhadra's attitude questionnaire (1966) includes the item "Have you ever known in advance that you are going to receive a particular letter on a particular day?" It is now possible to obtain this information through normal sensory means (digitally/online). Finally, many published instruments include items that refer to nonpsi phenomena such as astrology, the Loch Ness monster, heaven and hell, aliens/UFOs, and drug use (e.g., Gallagher et al., 1994; Jones et al., 1977; Otis \& Alcock, 1982; Randall \& Desrosiers, 1980).

As cultural norms change, acceptable language is adjusted, psi research findings become more widespread, and psi phenomena are appropriately portrayed as prevalent in the popular culture, research instruments will need to follow suit to be useful. The aim of the current project was to develop a survey instrument that could collect data from an experience-centered perspective. This is in line with the recommended guidelines for researchers (Moreira-Almeida \& Lotufo-Neto, 2017) for developing and refining surveys "in a more comprehensive and reliable way" including distinguishing the lived experience from its interpretations and adopting a neutral but empathetic attitude (p. 287). Moreira-Almeida and Lotufo-Neto also recommended that terminology be carefully chosen; that terms with causal or theoretical implications be avoided and, instead, that phenomenological descriptions be used.

Established survey development and piloting methods (Andrews et al., 2003; Dillman et al., 2014) were used to create the Windbridge Psi and Related Phenomena Awareness Questionnaire (WPRPAQ) which phenomenologically describes experiences without using potentially loaded or controversial terms. For each described experience, the respondent signifies whether they are aware of that phenomenon or not and, if they are, what experience they have had with it.

The WPRPAQ is a novel, web-based instrument. Web and mobile surveys are becoming increasingly common modalities for providing instruments to respondents (Dillman et al., 2014). The benefits of electronic surveys (Andrews et al., 2003) include increased feasibility; 
cost effectiveness; quick distribution and response cycles; design principles similar to those of paper questionnaires; applicability of both open-ended and forced-choice items; formatting control; and enhanced survey presentation including colors, graphics, and animations. However, the nature of the Internet prevents random sampling, and nonresponse rates cannot be assessed. In addition, "economics, age, and ethnicity continue to produce significant gaps between online and offline populations" (Andrews et al., 2003, p. 190).

The WPRPAQ was initially used to collect data from self-identified mediums and non-mediums. Although it is possible for anyone to experience communication from the deceased and this experience has been reported across cultures since antiquity, a medium is someone who has this experience regularly, reliably, and often on-demand. During a modern mediumship reading, a medium shares information about and messages from the deceased with sitters, the living friends and loved ones of the deceased, for the purpose of generating for the sitters assisted after-death communication experiences (aADCs), one of the four types of ADCs (Beischel, 2019). Contemporary mediumship studies (reviewed in Beischel \& Zingrone, 2015) have examined the ability of mediums to report accurate and specific information about the deceased under controlled laboratory conditions (Beischel et al., 2015) as well as their psychological (Roxburgh \& Roe, 2011; Taylor \& Murray, 2012) and physiological (Beischel et al., 2019) characteristics and their specific experiences of communication with the deceased (e.g., Beischel et al., 2017; Emmons \& Emmons, 2003; Rock \& Beischel, 2008).

\section{METHODS}

Addressing the limitations of previous instruments discussed above, we developed the 10-item, WPRPAQ instrument (see Appendix) and collected online responses from self-identified mediums and nonmediums. This occurred as part of a larger online survey project termed the Online Census of Traits and Observations (OCTO) Study. Other OCTO Study findings are reported elsewhere.

\section{WPRPAQ Instrument}

An online questionnaire hosting and development service (i.e. FormSite) was utilized to create and host the WPRPAQ survey items 
and capture participant responses. Potential respondents were initially sent to a descriptive page on the Windbridge Institute website with links to the actual survey. The 52 OCTO Study items were completed by respondents in one sitting. No WPRPAQ items were required; initial survey items regarding participation requirements were necessary in order to submit a survey. OCTO Study data were collected during 2016 and 2017.

Piloting. The survey was developed using an established 4-stage survey piloting process (Andrews et al., 2003) which included (1) review by knowledgeable analysts (colleagues in or familiar with the field of parapsychology/psi-research) to ensure question completeness, efficiency, relevancy, scale, and format appropriateness; (2) "typical" participants (i.e. Windbridge Certified Research Mediums, WCRMs; Beischel, 2007) taking the survey while giving real-time as well as retrospective feedback to the investigators; (3) examination of the survey language, question interpretation consistency, and logical sequencing by employing a small number of pilot respondents from the general population; and (4) a final check to catch any inadvertently introduced typos or errors. Pilot data were collected from roughly 50 mediums and non-mediums prior to formal data collection. This piloting process established face validity to the instrument. Further, because the questions describe the phenomena of interest and ask if the respondent has ever heard of them, test/re-test reliability cannot be assessed since the process of taking the survey facilitates awareness. In addition, because each WPRPAQ item refers to an independent phenomenon, internal consistency reliability statistics are not appropriate and were not calculated.

Language. The WPRPAQ was designed to not include any terms (such as telepathy, clairvoyance, or reincarnation) that may be associated with respondents' biases or assumptions. This is similar to Palmer's (1979) psychic experiences survey in which the "primary questions were phrased as precise descriptions of the experience or activity of interest, using the simplest words possible. In most cases, we avoided the use of labels ... that might have different connotations for different respondents" (p. 224). In addition, the language used in the WPRPAQ does not include potentially offensive or disparaging qualifying terms such as alleged, purported, supposed, or reported to 
describe participants' experiences. Though it is not possible to explain these types of experiences within the current mainstream materialist scientific paradigm, they "have been defined as real and important" by those having them and thus "are real in their consequences" (Nowatzki \& Grant Kalischuk, 2009, p. 93). The WPRPAQ takes a neutral stance on the veridical nature of these experiences and instead just attempts to determine their prevalence.

Phenomena. Each WPRPAQ item describes an experience (most items begin with, "Some people report/experience ... ") and then asks the respondent about both their awareness of and their experience with that phenomenon. Because these are all experiences that have been reported by mentally healthy individuals, questioning participants' beliefs regarding whether or not these phenomena exist is not relevant and was not included.

Participants could choose responses asserting that they have never heard of the phenomenon, that they have heard of it but not experienced it, that they have heard of it but are unsure if they have experienced it, or that they have heard of it and experienced it. "Prefer not to say" was also an option for each item. Without using these terms, the WPRPAQ asks about: energy healing, mediumship, telepathy, clairvoyance/remote viewing (RV), micropsychokinesis (microPK), macropsychokinesis (macroPK), out-of-body experiences (OBEs), neardeath experiences (NDEs), children's memories of previous lives, and precognition.

Items were numbered 1-10, and possible responses were labeled a, b, c, etc., for each item. The online hosting service allowed a participant to choose one radio button-associated response for each item within the WPRPAQ. The WPRPAQ was not named in the online questionnaire and was simply labeled "Section 7 " within the OCTO Study. For use by other researchers, we recommend it be labeled by its acronym alone if a title is required.

The full WPRPAQ instrument is reproduced in the Appendix. It should be noted that \#8 (NDEs) addresses only NDEs that occur concurrent with clinical death. Though NDEs can occur outside of this situation, the WPRPAQ was designed only to capture this specific type. It should also be noted that item \#9 (past-life memories) has been changed from the version used during the data collection described 
here. It originally read, "Some people (usually children) experience memories, preferences, behaviors, and other characteristics that are associated with a different person who lived at an earlier time and died before they were born." Because of the notable percentage of people reporting this experience ("I have had specific memories from a previous life"), it is likely that participants were including in their responses pastlife, regression-associated memories of previous lives and/or other memories acquired during adulthood. As such, that item was changed to the following: "Some young children report experiencing memories, preferences, behaviors, and other characteristics that are associated with a different person who lived at an earlier time and died before they were born." The relevant response is now phrased: "As a young child, I experienced and reported to others my specific memories of a previous life."

\section{Participants}

Anonymous survey responses were collected from U.S. Internet users with a general interest in mediumship and related topics. Survey respondents were recruited via Windbridge Institute's website, email lists, and social media to include self-identified mediums and nonmediums. A request was made to other organizations with access to similar populations of interest (e.g., Rhine Research Center) to post the call for participation. The survey used disclosure information and no identifying participant information was collected.

Demographics. In the 8-section OCTO Study, Section 1 included items about the participants' basic demographic characteristics. Respondents listed their age in years.

For race, they were asked "How would you describe yourself? (Choose one or more from the following racial groups.)" The options listed were:

American Indian or Alaska Native [a person having origins in any of the original peoples of North and South America (including Central America), and who maintains a tribal affiliation or community attachment]

Asian (a person having origins in any of the original peoples of the Far East, Southeast Asia, or the Indian subcontinent 
including, for example, Cambodia, China, India, Japan, Korea, Malaysia, Pakistan, the Philippine Islands, Thailand, and Vietnam)

Black or African American (a person having origins in any of the Black racial groups of Africa-includes Caribbean Islanders and others of African origin)

Native Hawaiian or Other Pacific Islander (a person having origins in any of the original peoples of Hawaii, Guam, Samoa, or other Pacific Islands)

White (a person having origins in any of the original peoples of Europe, the Middle East, or North Africa)

Prefer not to say

At the time of data collection, the U.S. government defined Hispanic as an ethnicity, not a race, so it was not an option on government forms and was not included in the OCTO Study. However, in the 2020 U.S. census, racial groups will be called "categories" and Hispanic will be included as an option (Cohn, 2015). For researchers wishing to collect this kind of demographic data with WPRPAQ data, we recommend using an updated race item based on what will be used in the U.S. 2020 Census: "Which category best describes you? Check all that apply." The possible options are

American Indian or Alaska Native (of, for example, Aztec, Navajo, Mayan, etc., descent)

Asian (of, for example, Asian Indian, Chinese, Filipino, Japanese, Korean, Vietnamese, etc., descent)

Black or African American (of, for example, African, Haitian, Jamaican, etc., descent)

Hispanic, Latino, or Spanish origin (of, for example, Cuban, Dominican, Mexican, Puerto Rican, Salvadoran, etc., descent)

Middle Eastern or North African (of, for example, Algerian, Egyptian, Lebanese, Moroccan, Persian, Syrian, etc., descent)

Native Hawaiian or Pacific Islander (of, for example, Fijian, Native Hawaiian, Samoan, Tongan, etc., descent)

White (of, for example, English, French, German, Italian, Irish, Polish, Russian, etc., descent) 
Another race, ethnicity, or origin

Prefer not to say

In Section 1, the OCTO Study participants were also asked, "With what gender do you currently identify yourself?" Response options were:

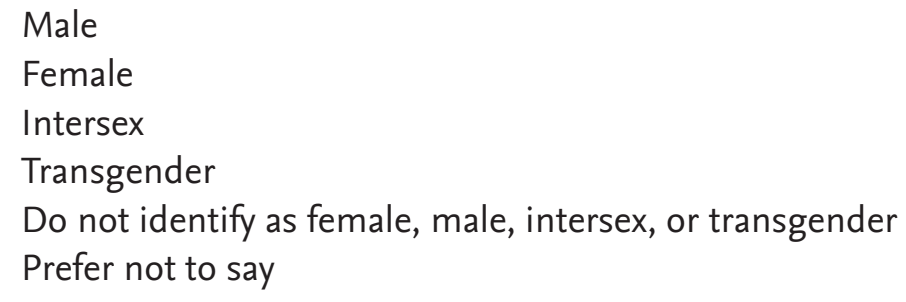

Identification as mediums. Two items in the OCTO Study were used to separate the respondents' data into medium and non-medium groups: a yes/no item and a checkbox item. In Section 2 of the survey, the single item read:

Please read the following description and then answer the item honestly. In our research, we use the word 'medium' to describe a person who regularly experiences communication from the deceased and reports the information s/he receives to the living. According to this definition, are you a medium?

The possible choices were: 'Yes,' 'No,' and 'Prefer not to say.'

Section 8 of the OCTO Study survey included this introductory statement: "The following items ask about your individual perceptions, reactions, experiences, and feelings." One item asked, "Do any of the following statements apply to you? (Check all that apply.)" One of the choices listed was "I consider myself a medium."

For data analysis, in order to be considered a medium, a participant needed to answer 'Yes' to the yes/no item and also check the "I consider myself a medium" box. In order to be considered a non-medium, a participant needed to do the opposite: answer 'No' to the yes/no item and also not check the box. Participants who preferred not to answer the first item were not included in the study. 


\section{Analyses}

Online statistical calculators (www.socscistatistics.com, vassarstats.net) and Microsoft Excel (v. 2016) were used for statistical data analyses. Data are reported as mean \pm standard deviation (n.s. = not significant). Bonferroni correction for multiple comparisons [30: 10 awareness, 10 experience, 10 blanks (see below)] resulted in an $\alpha$ of 0.0017 .

For each of the 10 WPRPAQ items, $1 \%$ or fewer of each sample left the item blank or chose "Prefer not to say." There were no significant differences between medium and non-medium samples for these proportions of these 'blanks' for any item (all $p \geq 0.02$ ).

WPRPAQ items were categorized as describing either bidirectional or unidirectional experiences. Bidirectional experiences involve two or more people and can be given and/or received by the experiencer/ respondent (energy healing, mediumship, and telepathy; items 1-3). Unidirectional experiences generally involve only the experiencer/ respondent (clairvoyance, microPK, macroPK, OBE, NDE, memories of previous lives, precognition; items 4-10).

Participant WPRPAQ responses were categorized according to awareness (yes or no) and experience (no, unsure, yes) for each of the 10 items. For example, item I describes the bidirectional phenomenon of energy healing and asks, "Were you aware that this type of healing exists?" Possible responses were:

a. No, l've never heard of this.

b. Yes, I have heard of this but never experienced it myself.

c. Yes, I have heard of this but I'm not sure if I've experienced it.

d. Yes, I have heard of this and someone else has given this type of healing to me.

e. Yes, I have heard of this and I have given this type of healing.

f. Both (d) and (e) are true for me.

Awareness. Awareness of a phenomenon was assessed by tallying participants who chose the 'no' response (a: 'No, l've never heard of this') and participants who chose any of the 'Yes' responses (in the example above: b, c, d, e, or f: 'Yes, I have heard of this ...').

For the awareness data, reported percentages are the proportions 
out of the total number of participants in a sample who chose an option ('blanks' removed). Comparisons of awareness for medium and non-medium samples were compared using z-score tests for two population proportions.

Experience. Experience with a bidirectional phenomenon (items 1-3) was assessed by tallying participants who had heard of a phenomenon and chose the 'none' response (b: 'never experienced it myself'), the 'unsure' response (c: 'I'm not sure if I've experienced it'), the 'received' response (d: 'someone else has done this for me'), the 'given' response (e: 'I have done this for someone else'), or the 'both' response (f: "Both (d) and (e) are true for me").

Experience with a unidirectional phenomenon (items 4-10) was assessed by tallying participants who had heard of a phenomenon and chose the 'no' response (b: 'never experienced it myself'), the 'unsure' response (c: 'I'm not sure if I've experienced it'), or the 'yes' response (d: I have experienced it).

Our research question involved comparing the experiences of participants in each group who were aware of the phenomena; thus, 'no' awareness responses were not included in the experience data analysis. For research questions involving the overall proportion of participants who never had an experience, tallying all the participants who chose either the 'no' awareness response (a) or the 'none' experience response (b) together would be appropriate.

For the experience data, reported percentages are the proportions out of the total number of participants in a sample who were aware of a phenomenon and who chose an experience option ('blanks' removed). Comparisons of experiences for medium and non-medium samples were compared using chi-squared contingency tables.

\section{RESULTS}

After all incomplete surveys, duplicates, and respondents who preferred not to answer the yes/no item were removed and the checkbox criteria were deployed (see METHODS: Participants: Identification as mediums), the remaining WPRPAQ data collected from 316 mediums and 1,030 non-mediums were analyzed. 


\section{Demographics}

Overall, the medium and non-medium participants showed no statistically significant differences in demographic characteristics: age $(53.2 \pm 10.1$ and $53.9 \pm 11.9$, respectively; $t$-test: n.s.), race (both roughly $95 \%$ white; $z$-score: n.s.), and gender ( $89.5 \%$ and $85.5 \%$ female, respectively; z-score: n.s.).

\section{Awareness of Phenomena}

Table 1 conveys the proportion of medium and non-medium samples who reported being aware of each of the 10 WPRPAQ phenomena; that is, the percentage of participants in each group who chose responses including the phrase, "Yes, I have heard of this" out of the total number of participants once blanks and "Prefer not to say" responses were removed. More than four-fifths of each participant sample in this study reported having heard of all 10 phenomena.

Considering all 10 phenomena, the fewest number of participants in either group (but still more than $80 \%$ ) had heard of microPK or macroPK. However, the portion of mediums who reported having heard of microPK and macroPK was significantly larger than the portion of non-mediums reporting the same for those phenomena (each $p<$ $.00001)$. No differences between samples existed for awareness of any of the other eight phenomena.

\section{Experience of Phenomena}

Participants reported all levels of experience with each phenomenon (i.e. no, unsure, and yes, including given, received, and both).

Though the portions of participants in each group who were aware of 8 of the 10 phenomena were not statistically significantly different (Table 1), their levels of experiences with each of the 10 showed significant differences (Table 2). There were significant associations between the types of experiences examined and whether participants self-identified as mediums or non-mediums [energy healing: $\chi^{2}(4)=269.07$; mediumship: $\chi^{2}(4)=720.71 ; \chi^{2}(4)=$ telepathy: $\chi^{2}(4)=325.00$; clairvoyance/RV: $\chi^{2}(2)=278.97$; microPK: $\chi^{2}(2)=101.55$; macroPK: $\chi^{2}(2)=85.30$; OBE: $\chi^{2}(2)=258.75$; NDE: $\chi^{2}(2)=119.24$; past- 
TABLE 1

\section{Portion of Participants Who Reported} Being Aware of Each Phenomenon

\begin{tabular}{|c|c|c|c|c|c|c|c|c|c|c|}
\hline & 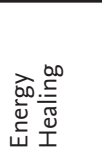 & 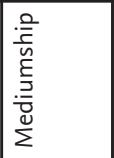 & 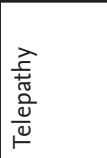 & 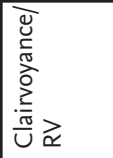 & 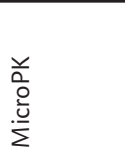 & 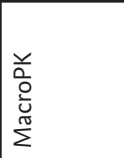 & 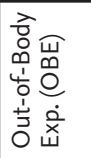 & 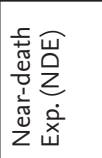 & 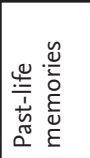 & 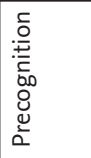 \\
\hline MED & $100.0 \%$ & $100.0 \%$ & $100.0 \%$ & $100.0 \%$ & $92.6 \%$ & $96.2 \%$ & $99.7 \%$ & $100.0 \%$ & $99.7 \%$ & $99.7 \%$ \\
\hline NON & $97.3 \%$ & $99.9 \%$ & $99.3 \%$ & $98.4 \%$ & $82.4 \%$ & $87.7 \%$ & $98.7 \%$ & $99.7 \%$ & $98.1 \%$ & $99.3 \%$ \\
\hline$z$ & 2.9645 & 0.5554 & 1.4661 & 2.2194 & 4.4188 & 4.3367 & 1.4530 & 0.9608 & 1.9608 & 0.7364 \\
\hline$p$ & n.s. & n.s. & n.s. & n.s. & $p<.00001$ & $p<.00001$ & n.s. & n.s. & n.s. & n.s. \\
\hline
\end{tabular}

life memories: $\chi^{2}(2)=204.75$; precognition: $\chi^{2}(2)=257.87$; all $\left.p<.0001\right]$. That is, a significantly larger portion of mediums than non-mediums had each of the 10 experiences. Effect sizes (Cramer's V; e.g., Kim, 2017) for the differences in experience between mediums and nonmediums were considered large for all three bidirectional phenomena (energy healing, mediumship, and telepathy; $d f=4 ; V>0.25$ ) and for the unidirectional phenomena of clairvoyance/RV, OBEs, past-life memories, and precognition ( $d f=2 ; V>0.35)$. Effect sizes were medium for the unidirectional phenomena of NDEs and microPK and macroPK $(d f=2 ; V>0.21)$.

Bidirectional phenomena. For experiences with the bidirectional phenomena of energy healing, mediumship, and telepathy (Figure 1), more than $60 \%$ of mediums had both given and received each experience $(63.2 \%, 78.4 \%$, and $76.8 \%$, respectively). More than $20 \%$ of non-mediums reported never having experienced each of these bidirectional phenomena (35.5\%, 20.2\%, and $22.0 \%$, respectively). Roughly one-fifth of non-mediums were unsure if they had experienced each of these three $(20.2 \%, 19.5 \%$, and $22.5 \%$, respectively).

More than half of non-mediums in this study reported experience with acting as sitters: $56.8 \%$ reported that "someone else has described accurate information about a deceased person to me" (received + both).

Unidirectional phenomena. For the unidirectional basic psi phenomena of clairvoyance/RV (\#4), precognition (\#10), microPK (\#5), 
TABLE 2

Results of $\chi^{2}$ Tests Comparing Experiences of Medium and Non-medium Participants

\begin{tabular}{lcccl}
\hline \hline & $\chi^{2}$ & $d f$ & $p$ & Cramer's V \\
\hline Energy Healing & 269.07 & 4 & $<.0001$ & 0.4529 \\
Mediumship & 720.71 & 4 & $<.0001$ & 0.7345 \\
Telepathy & 325.00 & 4 & $<.0001$ & 0.4934 \\
Clairvoyance/RV & 278.97 & 2 & $<.0001$ & 0.4587 \\
MicroPK & 101.55 & 2 & $<.0001$ & 0.2992 \\
MacroPK & 85.30 & 2 & $<.0001$ & 0.2665 \\
OBE & 258.75 & 2 & $<.0001$ & 0.4412 \\
NDE & 119.24 & 2 & $<.0001$ & 0.2985 \\
Past-life memories & 204.75 & 2 & $<.0001$ & 0.3938 \\
Precognition & 257.87 & 2 & $<.0001$ & 0.4400 \\
& & & & \\
\hline
\end{tabular}

$\mathrm{RV}=$ Remote viewing. $\mathrm{PK}=$ Psychokinesis. $\mathrm{OBE}=$ Out-of-body experience . $\mathrm{NDE}=$ Near-death experience. Bonferroni corrected $\alpha=0.0017$.

and macroPK (\#6)(Figure 2), 40\% or more of non-mediums had no such experiences ("never experienced it myself;" 42.5\%, 46.9\%, 66.7\%, and $72.2 \%$, respectively). Roughly $80 \%$ of mediums reported experiencing clairvoyance/RV or precognition ( $81.8 \%$ and $79.6 \%$, respectively). Fewer than $30 \%$ of non-mediums reported having these two types of psi experiences (29.6\% and $29.5 \%$, respectively).

Many participants in both samples reported either that they had never experienced microPK or macroPK phenomena or that they were unsure if they had or not (no + unsure-mediums: $79.6 \%$ for microPK and $81.8 \%$ for macroPK; non-mediums: $93.4 \%$ for microPK and $93.2 \%$ for macroPK).

Two of the unidirectional phenomena assessed by the WPRPAQ are related to the concept of the continuation of consciousness after death (survival): NDEs (\#8) and past-life memories (\#9). The majority of mediums and non-mediums reported never having experienced an NDE (62.9\% and $88.7 \%$, respectively); $17.5 \%$ of mediums and $3.6 \%$ 


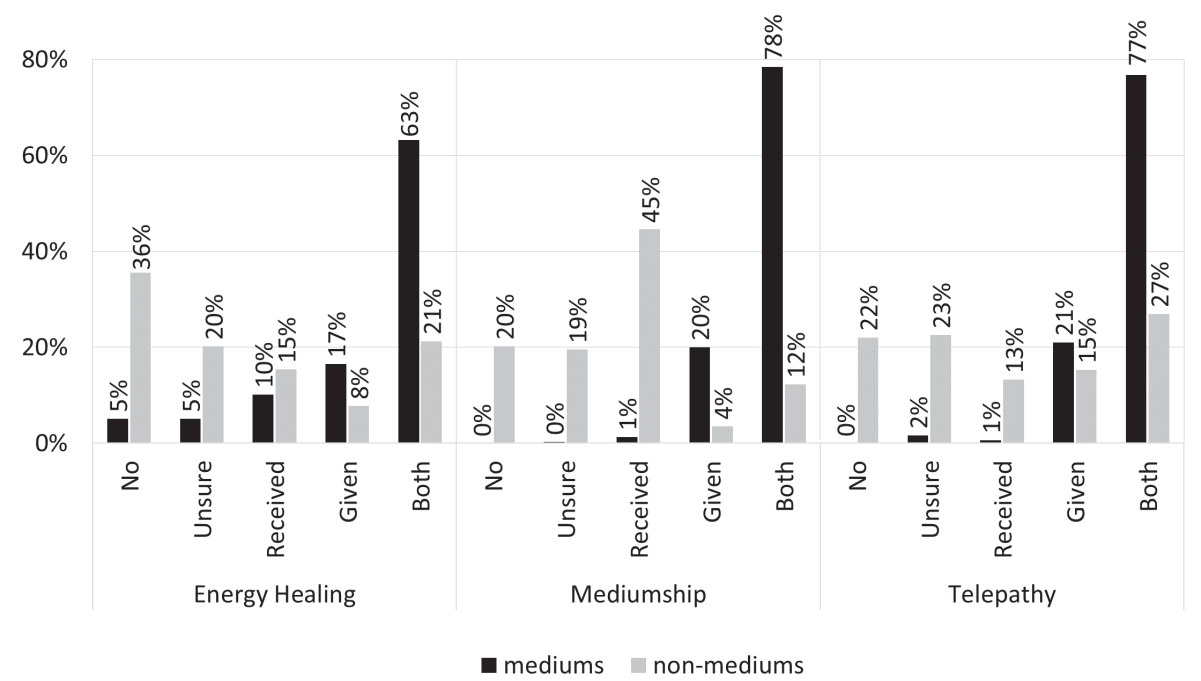

Figure 1. Levels of experience with bidirectional psi-related phenomena in selfidentified mediums and non-mediums.

$\chi^{2}$ tests: all $p<.0001 ; d f=4$; Cramer's $V>0.25$; Bonferroni corrected $\alpha=0.0017$ (see Table 2). No = "never experienced it myself." Unsure = "I'm not sure if I've experienced it." Both = received and given. Energy Healing: Received = "someone else has given this type of healing to me." Given = "I have given this type of healing." Mediumship: Received = "someone else has described accurate information about a deceased person to me." Given = "I have described accurate information about deceased people I didn't know." Telepathy: Received: "someone else has acquired accurate information about me like this." Given = "I have acquired accurate information about someone else like this." For ease of viewing the graph, portions are rounded to the nearest whole number.

of non-mediums reported NDEs: having conscious experiences of themselves in a plane of existence different from the physical world they are used to "when I was clinically dead or close to it" (Figure 3).

The majority of mediums (53.7\%) and $15.7 \%$ of non-mediums reported having experienced past-life memories (Figure 3). However, these proportions may include memories experienced during adulthood and the item was rephrased after data collection to include only the phenomenon of children who remember past lives (e.g., Tucker, 2008; see METHODS: WPRPAQ Instrument: Phenomena). Twenty-three percent of mediums and $60.6 \%$ of non-mediums chose the option, 


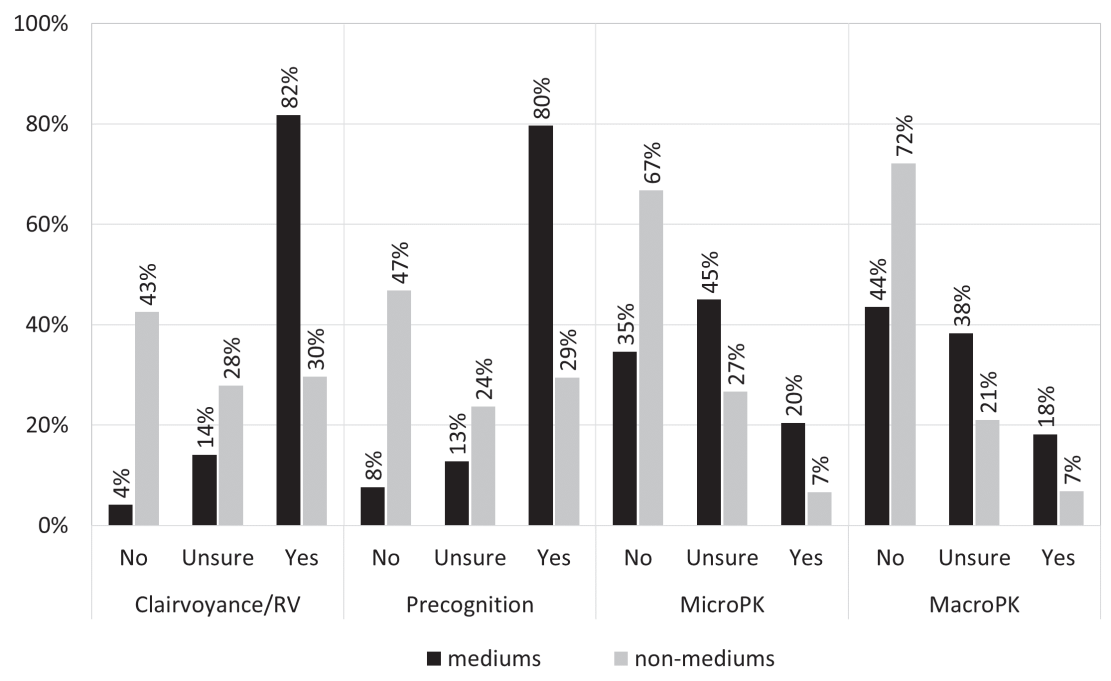

Figure 2. Levels of experience with unidirectional basic psi phenomena in selfidentified mediums and non-mediums.

$\chi^{2}$ tests: all $p<.0001$; Bonferroni-corrected $\alpha=0.0017$ (see Table 2). No $=$ "never experienced it myself." Unsure = "I'm not sure if l've experienced it." Clairvoyance: Yes = "I have acquired accurate information about a distant or concealed object or event like this." Precognition: Yes = "I have acquired accurate information about a future event that I couldn't have logically predicted." MicroPK: Yes = "I have affected a quantum-sized system using only my mind." MacroPK: Yes = "I have affected a largerthan-quantum-sized system using only my mind." For ease of viewing the graph, portions are rounded to the nearest whole number.

"never experienced it myself" related to past-life memories.

For the unidirectional phenomenon of OBEs (\#7), which is tangentially associated with survival, $78.4 \%$ of mediums and $28.3 \%$ of non-mediums reported "I have had experiences during which I felt that I was separate from my body." The proportions of mediums and non-mediums who reported never having had an OBE were $10.8 \%$ and $53.1 \%$, respectively (Figure 3).

\section{DISCUSSION}

It was expected that the majority of survey respondents in this study would be aware of most of the psi and related phenomena described, as these are the research interests of the organizations that recruited the participants. Similarly, Kohr (1980) found that, "Since ARE members 


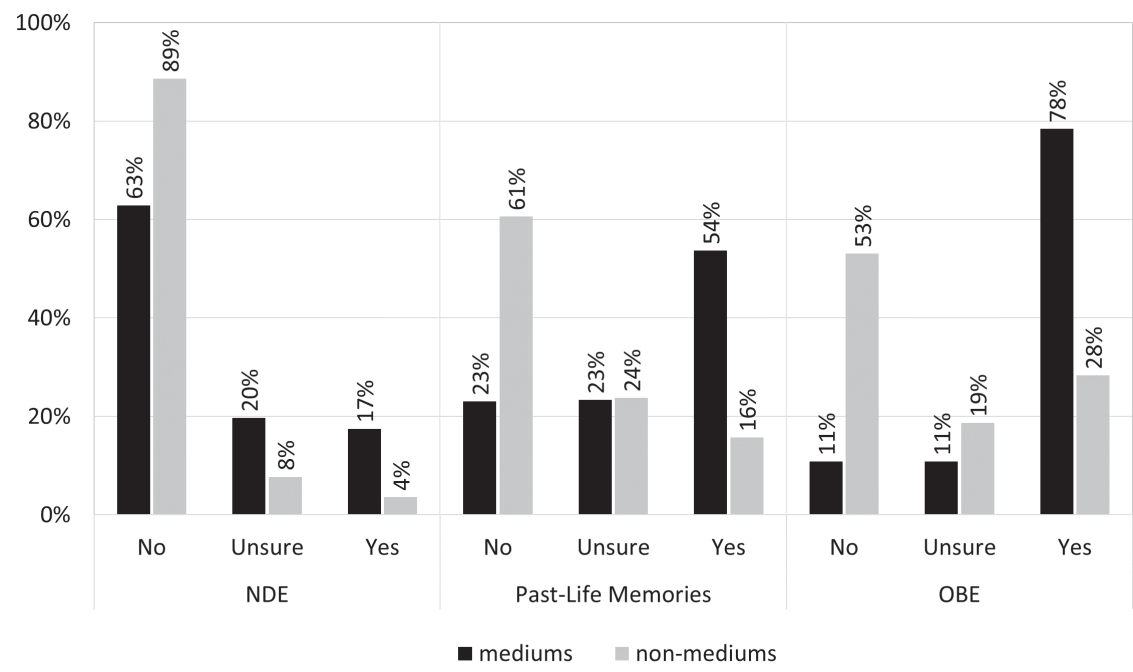

Figure 3. Levels of experience with unidirectional survival-related phenomena in self-identified mediums and non-mediums.

$\chi^{2}$ tests: all $p<.0001$; Bonferroni corrected $\alpha=0.0017$ (see Table 2). No = "never experienced it myself." Unsure = "I'm not sure if I've experienced it." Out-of-Body Experience: Yes = "I have had experiences during which I felt that I was separate from my body." Near-Death Experience: Yes = "I have had a conscious experience like this when I was clinically dead or close to it." Past-Life Memories*: Yes = "I have had specific memories from a previous life." For ease of viewing the graph, portions are rounded to the nearest whole number. * See Methods for a discussion regarding the wording of this item.

represent a special population of individuals attracted to such an organization because of their personal interest in psi, the high incidence of claimed psi experiences in the poll was not surprising" (p. 395). These types of results should not be expected when using the WPRPAQ with general populations.

MicroPK was the least known and the least experienced of any of the phenomena. This may be because these effects require specialized equipment (e.g., random number generators) and are observable only through statistical analysis. That the participants in this study $(92.6 \%$ of mediums and $82.4 \%$ of non-mediums) reported being aware of microPK speaks to the participants' association with science-based 
organizations and the success of these organizations in normalizing these types of phenomena and educating their audiences about them.

The differences in the levels of experiences for mediums and non-mediums when no differences in awareness existed is interesting. Significantly more mediums reported experiencing each of the 10 phenomena than did the non-mediums (all $p<.0001$ ). This suggests that the experience of mediumistic phenomena may be related to experiences of other psi phenomena or may make one more open to having and reporting those experiences.

It is unclear why the $1.6 \%$ of participants $(n=5)$ who reported identifying as mediums in the yes/no OCTO Study item ("In our research, we use the word 'medium' to describe a person who regularly experiences communication from the deceased and reports the information s/he receives to the living. According to this definition, are you a medium?") chose the 'unsure' option or only the 'received' option when asked about their experience in the WPRPAQ item describing mediumship (\#2). This does not appear to be a critical portion of the sample in this study, but researchers may wish to use this WPRPAQ item to assess the consistency of participants' reports and remove participants with conflicting responses. As this is an interesting subset that we wanted to discuss, these data were included in this study. Because many people have spontaneous experiences related to the deceased, it is understandable that $15.6 \%(n=161)$ of participants identifying as non-mediums chose options involving "I have described accurate information about deceased people I didn't know" for this item; most likely, they do not have this experience regularly or reliably.

The validity of our findings may have limitations. As stated above, the convenience sample used here to explore the usefulness of the WPRPAQ provided a skewed set of responses from participants who were expected to be aware of the phenomena of interest. The differences in captured levels of experience for each of the 10 phenomena between mediums and non-mediums in this highly aware, demographically uniform population, however, demonstrates the usefulness of this instrument in assessing differences. As stated above, though web and mobile surveys are becoming increasingly common, random sampling is not possible online, nonresponse rates cannot be assessed, and gaps exist between online and offline populations. In addition, a limitation 
exists for this study that plagues all self-report surveys: Researchers have no assurance that participants' responses reflect reality. The predominantly homogenous sample of white females over 40 in this study also prevents its findings from easily being extrapolated to other populations.

\section{CONCLUSIONS}

The WPRPAQ appears to be a useful survey instrument for collecting data from an experience-centered perspective. It assesses respondents' awareness of and experience with psi and related phenomena without overburdening participants, including interdependent items, relying on ideology or assumptions about the nature of the experiences or those who have them, using leading or other problematic terminology or language, or referring to phenomena not related to psi. Ideally, the WPRPAQ can be used by other researchers to assess awareness of psi and related phenomena and the prevalence of those experiences in other populations.

\section{ACKNOWLEDGMENTS}

This project was supported through grants from the Bial Foundation (\#372/14) and the Parapsychology Foundation.

\section{REFERENCES}

Andrews, D., Nonnecke, B., \& Preece, J. (2003). Electronic survey methodology: A case study in reaching hard-to-involve Internet users. International Journal of Human-Computer Interaction, 16(2), 185-210. https://doi.org/10.1207/ Si532759olJHC1602_04

Beischel, J. (2007). Contemporary methods used in laboratory-based mediumship research. Journal of Parapsychology, 71(1/2), 37-68.

Beischel, J. (2019, May). Spontaneous, facilitated, assisted, and requested afterdeath communication experiences and their impact on grief. Threshold: Journal of Interdisciplinary Consciousness Studies, 3(1): 1-32.

Beischel, J., Boccuzzi, M., Biuso, M., \& Rock, A. J. (2015, March-April). Anomalous information reception by research mediums under blinded conditions II: Replication and extension. EXPLORE: The Journal of Science \& Healing, 11(2), 136-142. https://doi.org/10.1016/j.explore.2015.01.001

Beischel, J., Mosher, C., \& Boccuzzi, M. (2017). Quantitative and qualitative analyses of mediumistic and psychic experiences. Threshold: Journal of Interdisciplinary Consciousness Studies, 1(2): 51-91. 
Beischel, J., Tassone, S., \& Boccuzzi, M. (2019, March-April). Hematological and psychophysiological correlates of anomalous information reception in mediums: A preliminary exploration. EXPLORE: The Journal of Science $Q$ Y Healing, 15(2), 126-133. https://doi.org/10.1016/j.explore.2018.04.009

Beischel, J., \& Zingrone, N. L. (2015). Mental mediumship. In E. Cardeña, J. Palmer, \& D. Marcusson-Clavertz (Eds.), Parapsychology: A handbook for the 21st century (pp. 301-313). McFarland.

Bhadra, B. H. (1966). The relationship of test scores to belief in ESP. Journal of Parapsychology, 30, 1-17.

Castro, M., Burrows, R., \&Wooffitt, R. (2014). The paranormal is (still) normal: The sociological implications of a survey of paranormal experiences in Great Britain. Sociological Research Online, 19(3), 1-15. https://doi.org/10.5153/sro.3355

Cohn, D. (2015, June 18). Census considers new approach to asking about race-By not using the term at all. Pew Research Center. https://www.pewresearch.org/fact-tank/2015/06/18/census-considersnew-approach-to-asking-about-race-by-not-using-the-term-at-all/

Dillman, D. A., Smyth, J. D., \& Christian, L. M. (2014). Internet, phone, mail, and mixed-mode surveys: The tailored design method. John Wiley \& Sons.

Emmons, C. F., \& Emmons, P. (2003). Guided by spirit: A journey into the mind of the medium. iUniverse.

Fach, W., Atmanspacher, H., Landolt, K., Wyss, T., \& Rössler, W. (2013). A comparative study of exceptional experiences of clients seeking advice and of subjects in an ordinary population. Frontiers in Psychology, 4(65), 1-10. https://doi.org/10.3389/fpsyg.2013.00065

Gallagher, C., Kumar, V. K., \& Pekala, R. J. (1994). The anomalous experiences inventory: Reliability and validity. The Journal of Parapsychology, 58(4), 402-428.

Goulding, A., \& Parker, A. (2001). Finding psi in the paranormal: Psychometric measures used in research on paranormal beliefs/experiences and in research on psi-ability. European Journal of Parapsychology, 16, 73-101.

Irwin, H. J. (1994). Childhood trauma and the origins of paranormal belief: A constructive replication. Psychological Reports, 74(1), 107-111. https://doi. org/10.2466/pro.1994.74.1.107

Irwin, H. J. (2009). The psychology of paranormal belief: A researcher's handbook. University of Hertfordshire Press.

Jones, W. H., Russell, D. W., \& Nickel, T. W. (1977). Belief in the paranormal scale: An objective instrument to measure belief in magical phenomena and causes. Journal Supplement Abstract Service, Catalog of Selected Documents in Psychology, 7, 100 (MS 1577).

Kennedy, J. E., Kanthamani, H., \& Palmer, J. (1994). Psychic and spiritual experiences, health, well-being, and meaning in life. Journal of Parapsychology, 58(4), $353-383$. 
Kim, H.-Y. (2017). Statistical notes for clinical researchers: Chi-squared test and Fisher's exact test. Restorative Dentistry \& Endodontics, 42(2), 152-155. https://doi.org/10.5395/rde.2017.42.2.152

Kohr, R. L. (1980). A survey of psi experiences among members of a special population. The Journal of the American Society for Psychical Research, 74(4), 395-411.

Moreira-Almeida, A., \& Lotufo-Neto, F. (2017). Methodological guidelines to investigate altered states of consciousness and anomalous experiences. International Review of Psychiatry, 29(3), 283-292. https://doi.org/10.1080/ 09540261.2017.1285555

Nixon, H. K. (1925, July). Popular answers to some psychological questions. The American Journal of Psychology, 36(3), 418-423. https://doi. org/10.2307/1414166

Nowatzki, N. R., \& Grant Kalischuk, R. (2009). Post-death encounters: Grieving, mourning, and healing. OMEGA: Journal of Death and Dying, 59(2), 91-111. https://doi.org10.2190/OM.59.2.a

Otis, L. P., \& Alcock, J. E. (1982). Factors affecting extraordinary belief. The Journal of Social Psychology, 118(1), 77-85. https://doi.org/10.1080/00224545.1982 .9924420

Palmer, J. (1979). A community mail survey of psychic experiences. Journal of the American Society for Psychical Research, 73(3), 221-251.

Rabeyron, T., \&Watt, C. (2010, March). Paranormal experiences, mental health and mental boundaries, and psi. Personality and Individual Differences, 48(4), 487-492. https://doi.org/10.1016/j.paid.2009.11.029

Randall, T. M. (1997). Paranormal short inventory. Perceptual and Motor Skills, 84(3_ suppl), 1265-1266. https://doi.org/10.2466/pms.1997.84.3c.1265

Randall, T. M., \& Desrosiers, M. (1980). Measurement of supernatural belief: Sex differences and locus of control. Journal of Personality Assessment, 44(5), 493-498. https://doi.org/10.1207/s15327752jpa4405-9

Rock, A. J., \& Beischel, J. (2008). Quantitative analysis of research mediums' conscious experiences during a discarnate reading versus a control task: A pilot study. Australian Journal of Parapsychology, 8(2), 157-179.

Roxburgh, E. C., \& Roe, C. A. (2011). A survey of dissociation, boundary-thinness, and psychological wellbeing in Spiritualist mental mediumship. Journal of Parapsychology, 75(2), 279-299.

Taylor, G., \& Murray, C. (2012). A qualitative investigation into non-clinical voice hearing: What factors may protect against distress? Mental Health, Religion \& Culture, 15(4), 373-388. https://doi.org/10.1080/13674676.2011. 577411

Thalbourne, M. A. (1995). Further studies of the measurement and correlates of belief in the paranormal. Journal of the American Society for Psychical Research, 89(3), 233-247.

Tobacyk, J. J. (2004). A revised paranormal belief scale. The International Journal of 
Transpersonal Studies, 23(1), 94-98. https://doi.org/10.1037/t14015-000

Tobacyk, J., \& Milford, G. (1983). Belief in paranormal phenomena: Assessment instrument development and implications for personality functioning. Journal of Personality and Social Psychology, 44(5), 1029-1037. https://doi. org/10.1037/0022-3514.44.5.1029

Tucker, J. B. (2008, July). Children's reports of past-life memories: A review. EXPLORE: The Journal of Science and Healing, 4(4), 244-248. https://doi.org/10.1016/j.explore.2008.04.001

Van de Castle, R. L., \& White, R. A. (1955). A report on a sentence completion form of sheep-goat attitude scale. Journal of Parapsychology, 19(3), 171-179.

\section{APPENDIX WPRPAQ Instrument}

The following items describe different phenomena that people have reported. For each item, read the statement and choose the option that best represents your personal experience.

1. Some people report the ability to-without the use of medications, surgery, or other physical treatments-send/receive healing to/from another person for the purpose of treating illness, injury, or other ailment or condition through the use of focused intention or other specific practice.

Were you aware that this type of healing exists?

a. No, l've never heard of this.

b. Yes, I have heard of this but never experienced it myself.

c. Yes, I have heard of this but I'm not sure if I've experienced it.

d. Yes, I have heard of this and someone else has given this type of healing to me.

e. Yes, I have heard of this and I have given this type of healing.

f. Both (d) and (e) are true for me.

Prefer not to say.

2. Some people experience and report to others communication from a deceased person that contains accurate and specific information and can occur without any prior knowledge about the deceased, without the use of any visual, verbal, or other feedback, and without using fraud.

Were you aware that people could provide information about the dead like this?

a. No, l've never heard of this.

b. Yes, I have heard of this but never experienced it myself.

c. Yes, I have heard of this but I'm not sure if I've experienced it.

d. Yes, I have heard of this and someone else has described accurate 
information about a deceased person to me.

e. Yes, I have heard of this and I have described accurate information about deceased people I didn't know.

f. Both (d) and (e) are true for me.

Prefer not to say.

3. Some people report knowing - without using any sensory cues (physically seeing, hearing, etc.)—accurate information about another person's thoughts or feelings.

Were you aware that this mind-only transfer of information exists?

a. No, I've never heard of this.

b. Yes, I have heard of this but never experienced it myself.

c. Yes, I have heard of this but I'm not sure if I've experienced it.

d. Yes, I have heard of this and someone else has acquired accurate information about me like this.

e. Yes, I have heard of this and I have acquired accurate information about someone else like this.

f. Both (d) and (e) are true for me.

Prefer not to say.

4. Some people report knowing-without using any sensory cues (physically seeing, hearing, etc.)-accurate information about an object or event that is at a distance or otherwise concealed from him/her.

Were you aware that this acquisition of information at a distance exists?

a. No, I've never heard of this.

b. Yes, I have heard of this but never experienced it myself.

c. Yes, I have heard of this but I'm not sure if I've experienced it.

d. Yes, I have heard of this and I have acquired accurate information about a distant or concealed object or event like this.

Prefer not to say.

5. Some people report being able to have an objective effect on tiny, quantumsized physical systems (such as random number generators) using only their minds.

Were you aware that this quantum effect of mind on matter exists?

a. No, l've never heard of this.

b. Yes, I have heard of this but never experienced it myself.

c. Yes, I have heard of this but I'm not sure if I've experienced it.

d. Yes, I have heard of this and I have affected a quantum-sized system using only my mind.

Prefer not to say. 
6. Some people report being able to have an observable effect on larger-thanquantum-sized physical systems (such as dice or other small objects) using only their minds.

Were you aware that this larger-than-quantum effect of mind on matter exists?

a. No, l've never heard of this.

b. Yes, I have heard of this but never experienced it myself.

c. Yes, I have heard of this but I'm not sure if I've experienced it.

d. Yes, I have heard of this and I have affected a larger-than-quantumsized system using only my mind.

Prefer not to say.

7. Some people report having experienced themselves (that is, their minds, awareness, or consciousness) as temporarily located separate from their physical bodies and able to observe their bodies and the surrounding environments.

Were you aware of this experience of being outside of the body?

a. No, l've never heard of this.

b. Yes, I have heard of this but never experienced it myself.

c. Yes, I have heard of this but I'm not sure if I've experienced it.

d. Yes, I have heard of this and I have had experiences during which I felt that I was separate from my body.

Prefer not to say.

8. Some people, on being revived after being clinically dead, report having had conscious experiences during the time that they were dead of themselves in a plane of existence different from the physical world they are used to.

Were you aware of the existence of these types of experiences?

a. No, l've never heard of this.

b. Yes, I have heard of this but never experienced it myself.

c. Yes, I have heard of this but I'm not sure if I've experienced it.

d. Yes, I have heard of this and I have had a conscious experience like this when I was clinically dead or close to it.

Prefer not to say.

9. Some young children report experiencing memories, preferences, behaviors, and other characteristics that are associated with a different person who lived at an earlier time and died before they were born.

Were you aware of the existence of these types of memories, etc., in young children?

a. No, l've never heard of this.

b. Yes, I have heard of this but, as a child, did not experience it myself.

c. Yes, I have heard of this but I'm not sure if I experienced it as a child. 
d. Yes, I have heard of this and as a young child I experienced and reported to others my specific memories of a previous life.

Prefer not to say.

10. Some people report knowing accurate information about an event that will happen in the future and that could not be logically predicted from current information.

Were you aware that this acquisition of information from the future exists?
a. No, l've never heard of this.
b. Yes, I have heard of this but never experienced it myself.
c. Yes, I have heard of this but I'm not sure if I've experienced it.
d. Yes, I have heard of this and I have acquired accurate information about a future event that I couldn't have logically predicted.
Prefer not to say. 